\title{
Enhanced TLR-induced NF-кB signaling and type I interferon responses in NLRC5 deficient mice
}

Yanzheng Tong ${ }^{1,2,3, *}$, Jun Cui ${ }^{1,3, *}$, Qingtian $\mathrm{Li}^{1,3}$, Jia Zou ${ }^{1,3}$, Helen Y Wang ${ }^{1,3,4}$, Rong-Fu Wang ${ }^{1,3,4}$

${ }^{I}$ The Center for Cell and Gene Therapy, Baylor College of Medicine, Houston, TX 77030, USA; ${ }^{2}$ Institute of Biosciences and Technology, Texas A\&M University Health Science Center, Houston, TX 77030, USA; ${ }^{3}$ Center for Inflammation and Epigenetics, The Methodist Hospital Research Institute, Houston, TX 77030, USA; ${ }^{4}$ Department of Pathology and Immunology, Baylor College of Medicine, Houston, TX 77030, USA

Nod-like receptors (NLRs) are intracellular sensors that respond to a variety of pathogen and intracellular danger signals to induce innate immune responses. NLRC5 has recently been identified to be an important regulator of $\mathrm{NF}-\kappa \mathrm{B}$, type I interferon (IFN) and inflammasome signaling pathways, but the in vivo function and mechanisms of NLRC5 remain to be defined. Here, we describe the generation and characterization of NLRC5 knockout mice. We show that induction of $N L R C 5$ expression by Toll-like receptor (TLR) ligand or cytokine stimulation requires the signal transducers and activators of transcription (Stat)1-mediated signaling pathway. NLRC5 ablation reduces MHC class I expression, and enhances IKK and IRF3 phosphorylation in response to TLR stimulation or viral infection. Consistent with these observations, we found that $N L R C 5$ deficiency enhanced IL-6 and IFN- $\beta$ production in mouse embryonic fibroblasts (MEFs), peritoneal macrophages and bone marrow-derived macrophages (BMMs), but not bone marrow-derived dendritic cells (BMDCs) after LPS stimulation or vesicular stomatitis virus (VSV) infection. Furthermore, we found that $N L R C 5$-deficient mice produced higher amounts of IL-6 and IFN- $\beta$ in the sera when they were challenged with LPS or infected with VSV. Taken together, these results provide in vivo evidence that NLRC5 plays critical roles in MHC class I expression, innate immune signaling and antiviral innate immune responses, thus serving as an important target for modulating innate immune signaling and regulation.

Keywords: innate immune signaling; NF- $\mathrm{kB}$ activation; type I interferon signaling; Nod-like receptors Cell Research (2012) 22:822-835. doi:10.1038/cr.2012.53; published online 3 April 2012

\section{Introduction}

The innate immune system is critically important as the first line of defense against invading pathogens by detecting or sensing pathogen- and danger-associated molecular patterns (known as PAMPs and DAMPs, respectively) through germline-encoded pattern recognition receptors (PRRs), which include Toll-like receptor (TLR) family, retinoic acid inducible gene I (RIG-I)like receptor (RLR) family, Nod-like receptor family and DNA sensors [1-4]. Upon PAMP stimulation, these PRRs trigger the activation of NF- $\mathrm{B}$, type I interferon

\footnotetext{
*These two authors contributed equally to this work.

Correspondence: Rong-Fu Wang

E-mail: rwang3@tmhs.org

Received 1 February 2012; revised 21 February 2012; accepted 24 February 2012; published online 3 April 2012
}

(IFN) or inflammasome signaling pathways, which leads to the production of proinflammatory and antiviral cytokines, and induction of subsequent adaptive immune responses against invading pathogens. Activation of most TLRs leads to the recruitment of a common adaptor, MyD88, and a series of downstream signaling events that culminate in NF- $\kappa \mathrm{B}$ activation and inflammatory responses. TLR3 recognizes viral dsRNA in endosomes and activates both NF- $\mathrm{KB}$ and type I IFN signaling pathways through TIR domain-containing adaptor-inducing interferon- $\beta$ (TRIF). RIG-I and MDA5 (melanoma differentiation-associated gene 5) function as cytoplasmic RNA sensors and recruit the mitochondrial protein called MAVS (also known as VISA, IPS-1 and Cardif) upon detection of intracellular RNA viruses [1-3]. Interferon- $\gamma$ inducible protein 16 (IFI16) and DEAD (Asp-Glu-AlaAsp) box protein 41 (DDX41) have been identified as cytosolic viral DNA sensors that activate the type I IFN 
signaling pathway [5-8].

NLRs represent a large group of protein family containing a conserved central nucleotide-binding and oligomerization domain (NOD), a leucine-rich repeat (LRR) region and a variable N-terminal effector domain [9]. While some NLRs, such as NOD1, NOD2 and NLRP3, serve as the PRRs in response to various PAMPs, others may function as negative regulators $[1,2,10]$. For example, NLRX1 has been shown to inhibit type I IFN signaling and $N F-\kappa B$ activation by interaction with MAVS or IKK $\alpha / \operatorname{IKK} \beta$ [11-13]. It is also implicated in the generation of reactive oxygen species [14]. NLRP4 has been reported to negatively regulate autophagic processes, NF- $\kappa \mathrm{B}$ and type I IFN signaling by interaction with Beclin, IKK $\beta$ and TBK1, respectively $[15$, 16]. More recently, NLRC5 has been identified as an important regulator of both innate and adaptive immune regulation [17-22]. While two groups have shown that NLRC5 inhibits NF- $\kappa \mathrm{B}$ and type I IFN signaling [17, 18], two other reports indicate that NLRC5 is required for IFN production in antiviral responses $[19,20]$. Furthermore, NLRC5 has been demonstrated to associate with NLRP3 to cooperatively activate the inflammasome [21]. NLRC5 has also been identified as a critical regulator of MHC class I gene expression [22]. Thus, it appears that NLRC5 plays diverse roles in innate immune regulation and MHC class I expression. To determine the in vivo function of NLRC5, Kumar et al. [23] generated $N L R C 5$-deficient mice by deleting exon 4 of NLRC5, but they did not observe any effect of NLRC5 ablation on proinflammatory cytokine production in response to virus and bacteria infection in bone marrow-derived dendritic cells (BMDCs). In this report, we describe the generation of $N L R C 5$-deficient mice by deleting exon 8 of NLRC5, and show that NLRC5 ablation enhanced $\mathrm{NF}-\kappa \mathrm{B}$ and type I IFN signaling pathways in response to various TLR stimulation or vesicular stomatitis virus (VSV) infection in multiple cell types, including mouse embryonic fibroblasts (MEFs), peritoneal macrophages and bone marrow-derived macrophages (BMMs), but not BMDCs. Furthermore, NLRC5-deficient mice produced higher amounts of IL- 6 and IFN- $\beta$ in the sera when they were challenged with LPS or infected with VSV, respectively. Taken together, these results demonstrate the critical role of NLRC5 in TLR-induced NF- $\kappa \mathrm{B}$ signaling and antiviral innate immune responses.

\section{Results}

Signal transducers and activators of transcription (Stat)1 is a key factor for NLRC5 induction

Although several groups have shown that NLRC5 can be induced by treatment with TLR ligands such as LPS, polyinosinic-polycytidylic acid (poly(I:C)), cytokines such as IFN- $\gamma$ and IFN- $\beta$, or virus infection, the mechanisms controlling its induction remain unclear [1720]. To further determine whether NLRC5 induction is regulated by NF- $\mathrm{KB}$ or IFN-mediated signaling pathway, we assessed NLRC5 expression in $M y D 88^{-/-}, I R A K 4^{-/-}$ or Stat $^{-/-}$mouse peritoneal macrophages in response to LPS or IFN- $\beta$ stimulation. $N L R C 5$ expression was

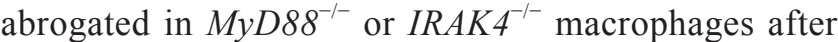
LPS treatment (Figure 1A), consistent with our previous report [17]. However, induction of NLRC5 expression in $M y D 88^{-/-}$or IRAK4 $4^{-1-}$ cells was identical to that in wildtype (WT) cells when they were treated with IFN- $\beta$, suggesting that induction of $N L R C 5$ expression by IFN- $\beta$ treatment remained intact in $M y D 88^{-/-}$or $I R A K 4^{-/-}$cells and is independent of NF- $\mathrm{KB}$ signaling. It is known that the Stat pathway plays a critical role in the expression of many cytokine-induced genes [24, 25]. Since LPS treatment can induce production of multiple cytokines, including IFN- $\beta$ and IFN- $\gamma$, we reasoned that induction of $N L R C 5$ expression by TLR ligands might result from the production of LPS-activated NF- $\mathrm{KB}$-responsive cytokines, which in turn induce NLRC5 expression. To test this possibility, we treated Stat ${ }^{-/}$peritoneal macrophages with LPS, IFN- $\gamma$ or IFN- $\beta$, and found that LPS-, IFN- $\gamma$ or IFN- $\beta$-mediated NLRC 5 induction was completely abrogated in Stat $^{-/}$peritoneal macrophages (Figure 1B). These data suggest that NLRC5 expression induced by LPS is dependent on Stat1 signaling. Furthermore, we found that the cell supernatants of LPStreated RAW264.7 cells were sufficient to induce NLRC5 expression (Supplementary information, Figure S1A and S1B). IFN- $\beta$ neutralizing antibody could almost completely abrogate LPS-mediated NLRC5 induction, while IFN- $\gamma$ neutralizing antibody had little effect (Supplementary information, Figure S1C and S1D), probably due to very low level of secreted IFN- $\gamma$ (data not shown). These results suggest that LPS-mediated NF- $\kappa \mathrm{B}$ activation leads to the production of IFN- $\beta$, which in turn induce NLRC 5 expression through the Stat1 signaling pathway.

NLRC5 deletion reduces MHC class I expression in T cells

To elucidate the physiological roles of NLRC5, we generated $N L R C 5$-deficient $\left(N L R C 5^{-1}\right)$ mice by homologous recombination in embryonic stem (ES) cells (Figure $2 \mathrm{~A}$ and $2 \mathrm{~B}$ ). The targeting vector was constructed by replacing a $2.1-\mathrm{kb}$ fragment, which encodes a functional LRR1 domain of NLRC5, with a Neo expression cassette (Figure 2A). The linearized targeting vector was then injected into ES cells by electroporation. Homologous recombinant stem cells were identified by screening with 
A

- WT Peritoneal Macrophages

A Myd88\% Peritoneal Macrophages
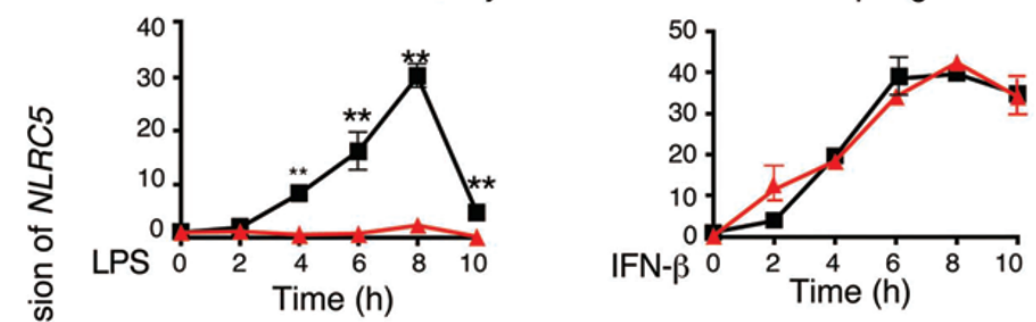

- WT Peritoneal Macrophages

$\Delta$ Irak4 \% Peritoneal Macrophages
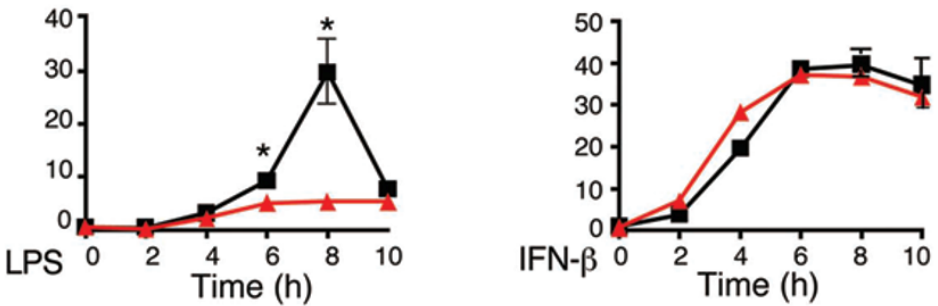

B

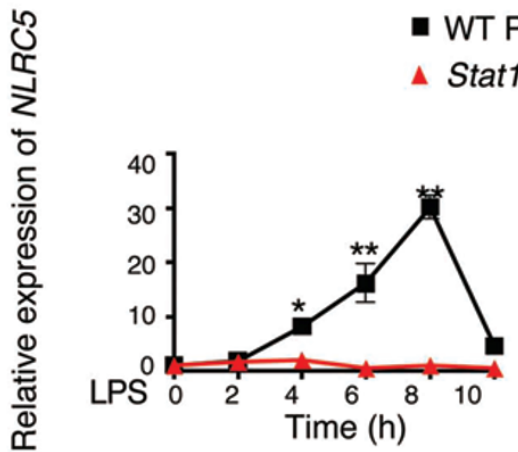

IFN-
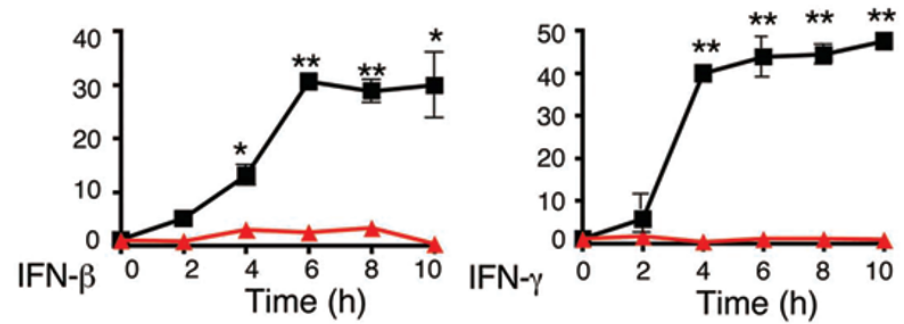

Figure 1 Stat1-mediated signaling pathway is critical for induction of NLRC5 expression. (A, B) Induction of NLRC5 expression by LPS $(100 \mathrm{ng} / \mathrm{ml})$, IFN- $\beta(1000 \mathrm{U} / \mathrm{ml})$ or IFN- $\gamma(10 \mathrm{ng} / \mathrm{ml})$ treatment in peritoneal macrophages from WT, $M y D 88^{-/}$, $I R A K 4^{-1-}(\mathrm{A})$ or Stat $1^{-1-}$ mice $(\mathrm{B})$. Results are representative of three independent experiments.

PCR and further confirmed by Southern blotting analysis (Supplementary information, Figure S2A and Figure 2C). The resultant recombinant products led to termination of NLRC5 open reading frame. The selected homologous recombinant ES clones were injected into blastocysts. Chimeric mice generated from two homologous ES clones were bred to generate F1 heterozygotes as well as homozygous NLRC5-deficient mice. NLRC5 knockout mice were born at a Mendelian ratio (Figure 2D) and appeared to be normal, compared with WT mice.

A recent study shows that overexpression of NLRC5 results in enhanced MHC class I expression in lymphoid as well as epithelial cell lines [22]. To assess the regulatory role of NLRC5 in MHC class I gene expression in vivo, we examined the expression of the mouse $\mathrm{MHC}$ class I molecule $\left(\mathrm{H} 2-\mathrm{K}^{\mathrm{b}}\right)$ in $\mathrm{CD} 4^{+} \mathrm{T}$ cells, $\mathrm{CD} 8^{+} \mathrm{T}$ cells and $\mathrm{B}$ cells isolated from NLRC5-deficient and WT mice. We found marked reduction of $\mathrm{H} 2-\mathrm{K}^{\mathrm{b}}$ in NLRC5-deficient $\mathrm{T}$ cells, compared to WT cells (Figure 2E). We also observed a small difference in MHC class I expression in NLRC5-deficient B cells compared with WT cells (Figure $2 \mathrm{E})$. In contrast, we observed little or no difference in MHC class II expression in NLRC5-deficient T cells and $\mathrm{B}$ cells (Figure 2E). It should be noted that MHC class II expression in NLRC5-deficient $\mathrm{CD} 8^{+} \mathrm{T}$ cells was a little higher than that in WT cells. These results suggest that NLRC5 is an important transcriptional regulator of MHC class I gene expression. 
NLRC5 ablation enhances TLR-induced NF- $\kappa B$ activation and proinflammatory cytokine production in $M E F S$

To investigate the role of NLRC5 in the NF- $\kappa \mathrm{B}$ path- way under physiological conditions, we treated MEFs generated from WT and NLRC5-deficient mice with LPS, followed by immunoblot analysis of phosphoryla-

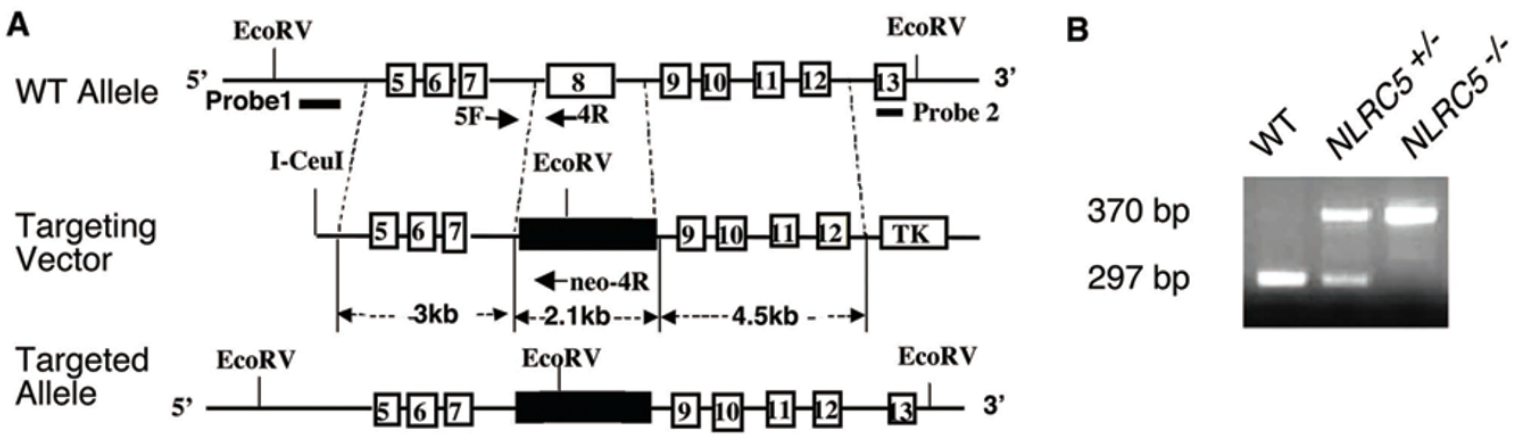

C

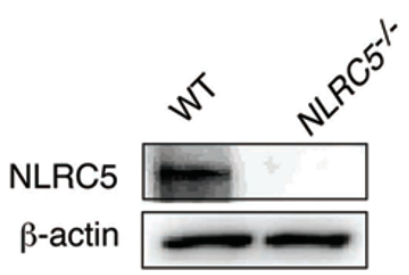

D

\begin{tabular}{lccc}
\hline NLRC5 & $\boldsymbol{+} / \boldsymbol{+}$ & $\boldsymbol{+ / -}$ & $-/-$ \\
\hline Total \# & 25 & 54 & 27 \\
$\%$ & 23.6 & 50.9 & 25.5 \\
\hline
\end{tabular}

E
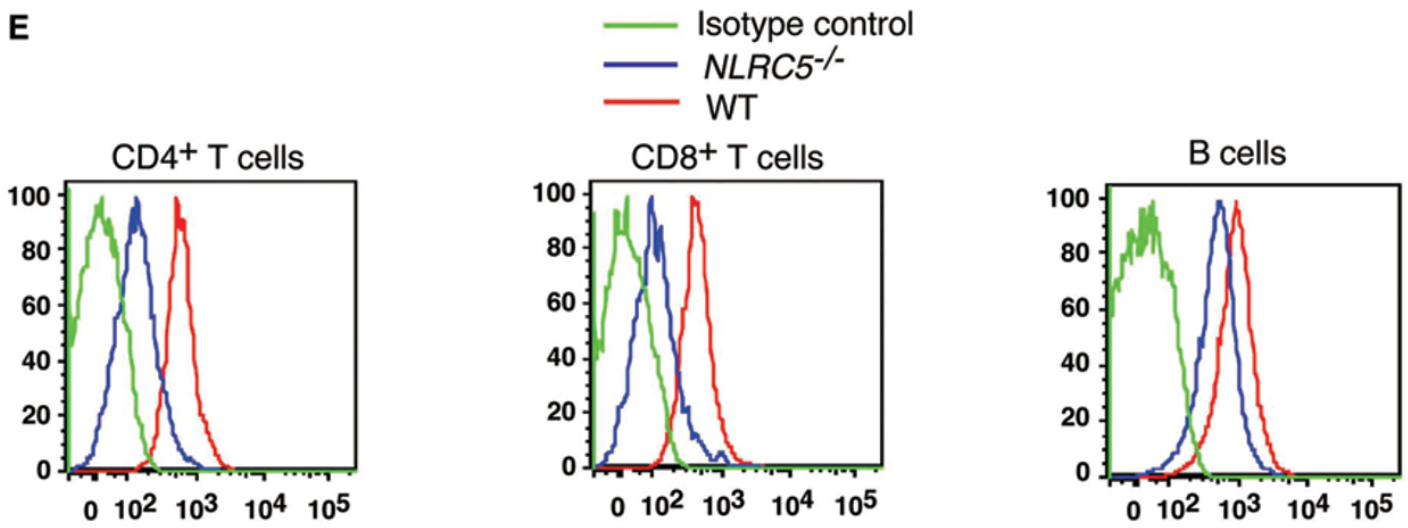

$\mathrm{H} 2-\mathrm{k}^{\mathrm{b}}$
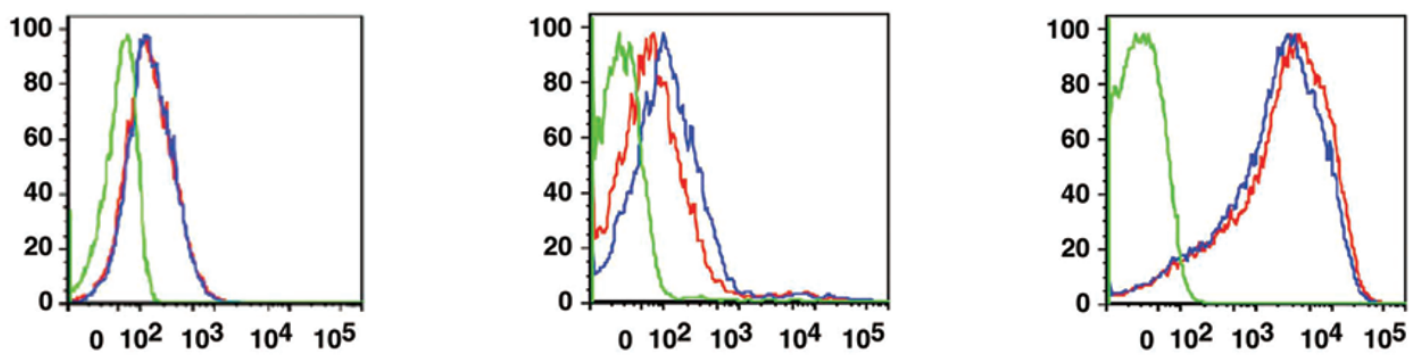

$1-A^{b}$

Figure 2 NLRC5 deletion reduces MHC class I expression. (A) Schematic presentation of generation of $N L R C 5^{-1-}$ mice through homologous recombination. (B) PCR genotyping of offspring from heterozygous breeding pairs. (C) Confirmation of the loss of NLRC5 protein in NLRC5 ${ }^{-1}$ T cells. (D) A Mendelian distribution of offspring from breeding of $N L R C 5^{+/}$mice. (E) $\mathrm{H} 2-\mathrm{K}^{\mathrm{b}}$ and $\mathrm{I}-\mathrm{A}^{\mathrm{b}}$ expression in WT and NLRC5-deficient CD4 ${ }^{+} \mathrm{T}$ cells, CD8 ${ }^{+} \mathrm{T}$ cells and $\mathrm{B}$ cells. Results are representative of three independent experiments. 
A

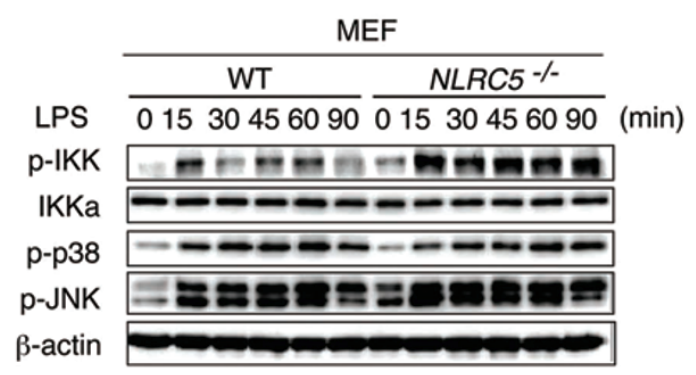

C WT MEF
ㅁ NLRC5 $\%$ MEF
B
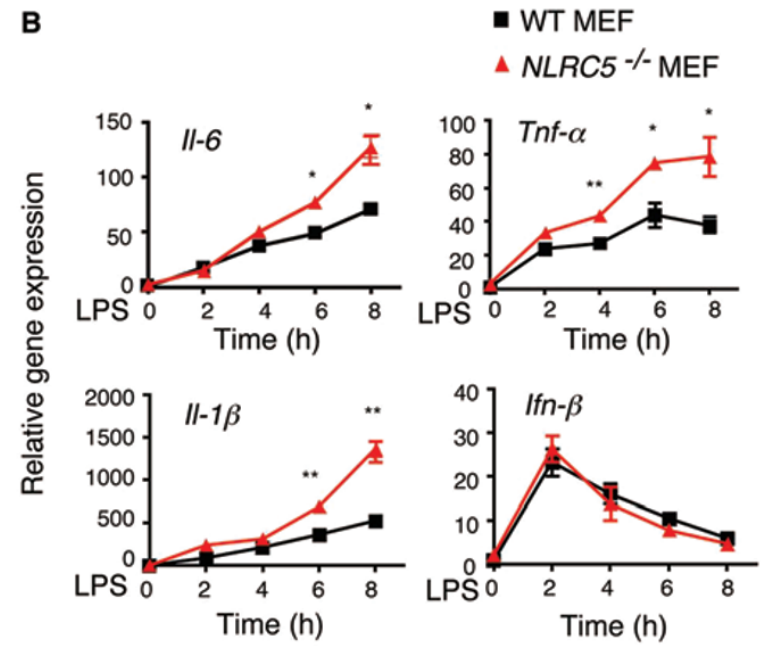
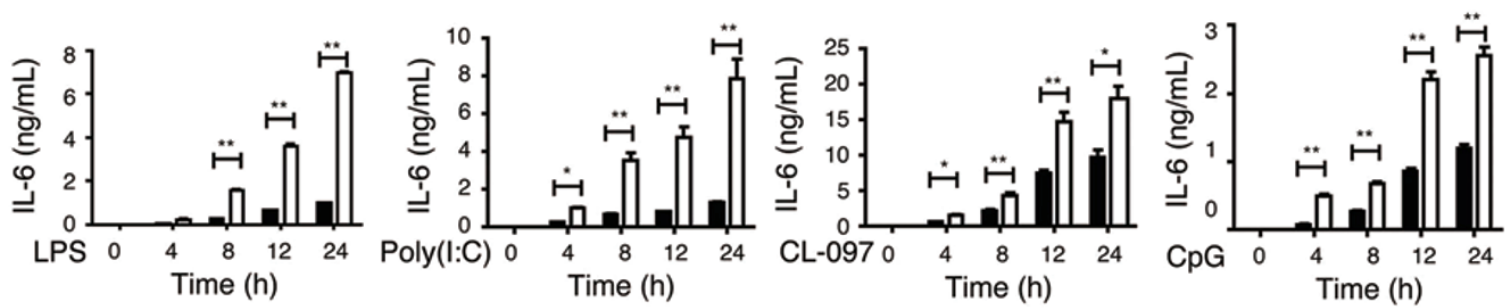

Figure 3 NLRC5 ablation markedly enhances NF-KB signaling and cytokine production in MEFs. (A, B) NLRC $5^{-1-}$ and WT MEFs were treated with LPS $(100 \mathrm{ng} / \mathrm{ml})$ for the indicated times, and the cell lysates were harvested for immunoblotting with the indicated antibodies (A) or RNA was collected for real-time PCR analysis (B). (C) MEF cells were incubated with LPS, poly(I:C), CL-097 or CpG for the indicated times, and the culture supernatants were used for measuring cytokines by ELISA. Data from $\mathbf{B}$ and $\mathbf{C}$ are plotted as means $\pm \mathrm{SD}$. Results are representative of three independent experiments. ${ }^{*} P<0.05$, ${ }^{* \star} P$ $<0.01$, versus controls.

tion of IKK and MAP kinases (p38 and JNK). We found that phosphorylation of IKK was markedly enhanced in $N L R C 5^{-1-}$ MEFs compared with WT MEFs after LPS stimulation. By contrast, we did not observe an appreciable difference in $\mathrm{p} 38$ and JNK phosphorylation between WT and $N L R C 5^{-/-}$MEFs (Figure 3A). To determine whether increased NF- $\mathrm{KB}$ activation in $N L R C 5^{-/}$cells correlates with expression of NF- $\mathrm{KB}$-responsive cytokine genes, we treated MEFs with LPS and then analyzed the expression of cytokines. The expression of $I L-6, T N F-\alpha$ and $I L-1 \beta$ in $N L R C 5^{-/-}$MEFs was significantly higher than in WT MEFs post LPS infection, but the mRNA level of Ifn- $\beta$, which is activated through the TLR4-TRIF pathway, was comparable between WT and $N L R C 5^{-/-}$ cells (Figure 3B). These results are consistent with data previously reported by our group and others $[17,18]$. Collectively, these results suggest that NLRC5 deletion enhances NF- $\mathrm{kB}$ activation as well as proinflammatory cytokine gene expression in MEFs following LPS stimulation.

We next determined the effect of NLRC5 deletion on
IL-6 and TNF- $\alpha$ production in MEFs following stimulation with TLR ligands, including poly(I:C), LPS, CL097 and CpG, which activate TLR3-, TLR4-, TLR7- and TLR9-mediated signaling pathways, respectively. We observed marked increases in IL-6 in $N L R C 5^{-/-}$MEFs compared to WT MEFs after stimulation with different TLR ligands (Figure 3C). TNF- $\alpha$ production was also increased in $N L R C 5^{--}$MEFs treated with LPS and $\mathrm{CpG}$, but not with poly(I:C), compared to that in WT MEFs (Supplementary information, Figure S3). Taken together, these results suggest that NLRC5 deletion enhances NF$\kappa \mathrm{B}$ activation and proinflammatory cytokine production in response to multiple TLR stimulation in MEFs.

\section{NLRC5 deficiency enhances antiviral immunity against} $R N A$ viruses in $M E F S$

To evaluate the effect of NLRC5 deficiency on antiviral innate immunity, we infected MEFs with a RNA virus, VSV-eGFP. IRF3 was phosphorylated as early as $4 \mathrm{~h}$ postinfection with VSV, and gradually increased with time. We found that the phosphorylation of IRF3 was 
at least twofold higher in $N L R C 5^{-/-}$MEFs, compared to that in WT MEFs by $8 \mathrm{~h}$ after viral infection (Figure 4A), while total IRF3 expression was comparable between the two groups. Moreover, loss of NLRC5 also increased the expression of type I IFN-responsive genes, including
$I F N-\alpha$ and $I F N-\beta$ after VSV-eGFP infection (Figure 4B). However, little or no differences in Tnf- $\alpha$ and Il-6 expression were detected (Figure 4B), consistent with our previous findings obtained from NLRC5-specific siRNA knockdown experiments [17]. Furthermore, ELISA anal-
A
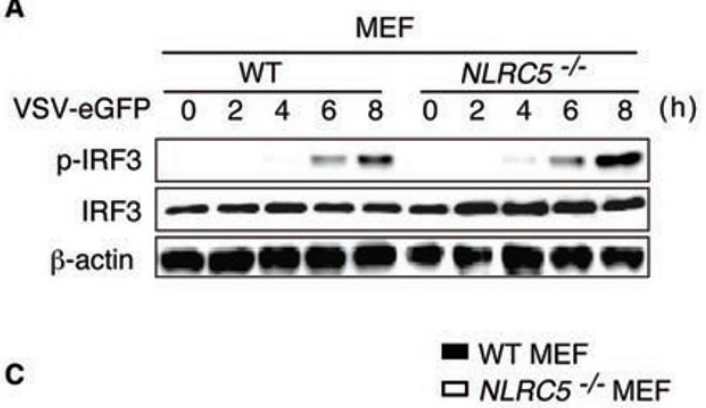
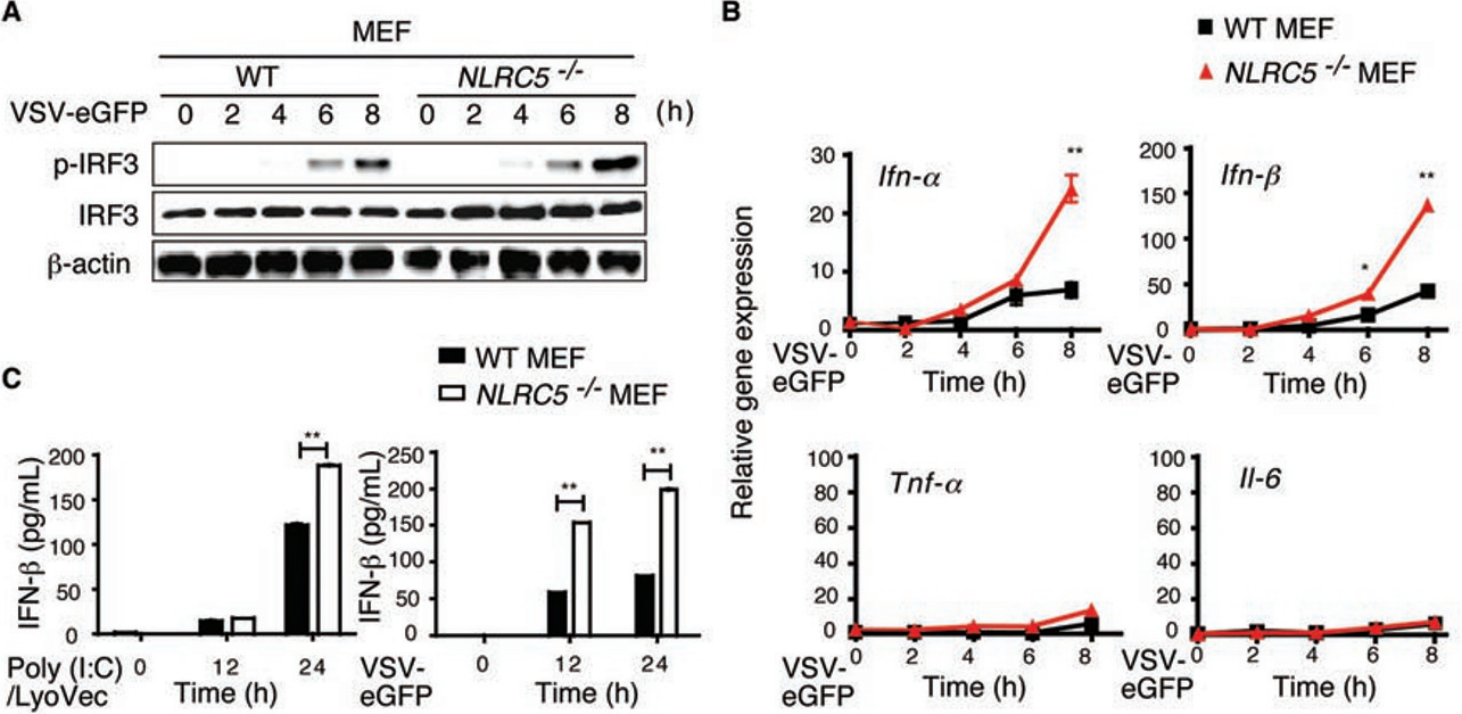

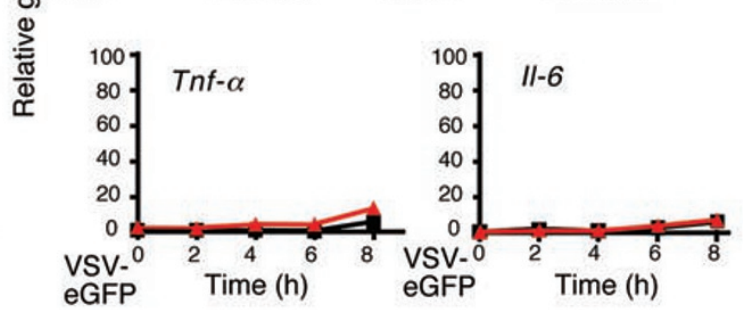

D

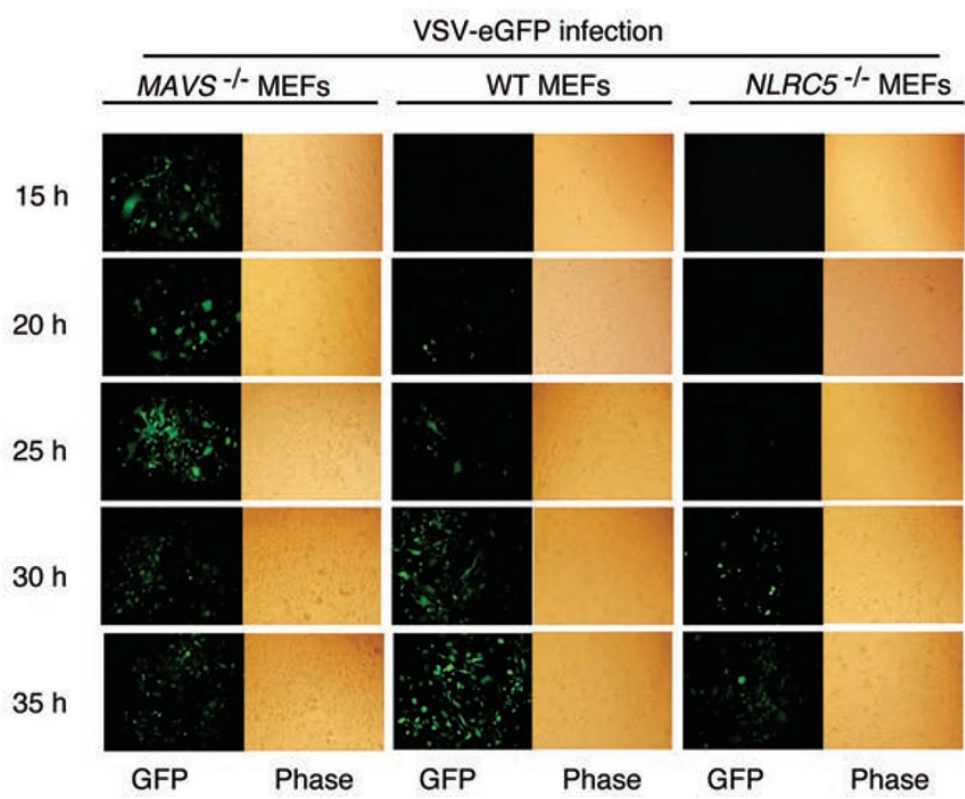

Figure 4 NLRC5 deficiency increases type I IFN signaling and antiviral immunity in MEFs. (A, B) NLRC5 $5^{-1-}$ and WT MEFs were infected with VSV-eGFP for the indicated times. Cell lysates were harvested to detect IRF3 phosphorylation by immunoblotting (A) or the RNA was collected to detect the expression of Ifn- $\alpha$, Ifn- $\beta$, II- 6 and Tnf- $\alpha$ by real-time PCR analysis (B). (C) MEFs were treated with poly(I:C)/LyoVec or infected with VSV-eGFP for the indicated times. The culture supernatants were harvested for ELISA analyses to measure the production of IFN- $\beta$. (D) MAVS ${ }^{-1}$, WT and $N L R C 5^{-1}$ MEFs were infected by VSV-eGFP $(\mathrm{MOI}=1)$ for the indicated times, viral infections were determined by fluorescence microscopy for GFP-positive cells and phase contrast as a control. $\mathbf{B}$ and $\mathbf{C}$ are plotted as means \pm SD. Results are representative of three independent experiments. ${ }^{*} P<0.05,{ }^{*} P<0.01$, versus controls. 
yses showed that intracellular poly(I:C) treatment (ligand for MDA5) or VSV-eGFP infection (ligand for RIG-I) strongly increased the secretion of IFN- $\beta$ in $N L R C 5^{-/}$ MEFs compared with that in WT MEFs (Figure 4C). These results suggest that NLRC5 deficiency enhanced IFN- $\beta$ activation through the RIG-I/MDA5 pathways.

To demonstrate a link between increased type I IFN response and antiviral immunity in $N L R C 5^{-/}$MEFs, we infected the $\mathrm{NLRC5}^{-/-}$, WT and $M A V S^{--}$cells with VSVeGFP $(\mathrm{MOI}=1)$, and infection was monitored based on GFP expression. GFP-positive (VSV-eGFP infected) cells could be detected at as early as $15 \mathrm{~h}$, peaked at $25 \mathrm{~h}$ and slightly decreased (due to cell apoptosis) at $30-35 \mathrm{~h}$ postinfection in $\mathrm{MAVS}^{-/}$cells (that were defective in type I IFN signaling) (Figure 4D). By contrast, we did not observe GFP-positive (VSV-eGFP infected) cells at $25 \mathrm{~h}$ in $N L R C 5^{-/-}$cells. The GFP-positive cells became detectable at $30 \mathrm{~h}$ and increased slightly at $35 \mathrm{~h}$ postinfection in $\mathrm{NLRC5}^{-/-}$cells (Figure 4D). WT MEFs showed intermediate GFP-positive cells (detectable at $20 \mathrm{~h}$ and gradually increased with time until $35 \mathrm{~h}$ postinfection; Figure 4D). Thus, $M A V S^{-1-}$ cells are more sensitive to VSVeGFP infection than WT MEFs, while NLRC5-deficient MEFs are more resistant to VSV-eGFP infection than WT cells, indicating that NLRC5 ablation increases the ability of MEFs to inhibit VSV infection, thus enhancing antiviral immunity.

NLRC5 negatively regulates $N F-\kappa B$ and type I IFN pathways in macrophages

To determine whether NLRC5 negatively regulates $\mathrm{NF}-\kappa \mathrm{B}$ and type I IFN pathways in immune cells, we performed similar experiments with BMDCs and found little or no difference in IL-6, TNF- $\alpha$ and IL-12 secretion in $\mathrm{NLRC5}^{-/-}$and WT BMDCs stimulated with various TLR ligands (Supplementary information, Figure S4A and S4B), which was consistent with a previous report showing that NLRC5 deficiency does not affect proinflammatory and type I IFN cytokines production in BMDCs and peritoneal exudates cells after stimulation with TLR agonists and RLRs [23]. However, when we treated peritoneal macrophages with LPS, we found that the phosphorylation of IKK was significantly increased in $N L R C 5^{-/-}$peritoneal macrophages, while the activation of MAP kinases (p38, ERK and JNK) was comparable between WT and $N L R C 5^{-/}$peritoneal macrophages (Figure $5 \mathrm{~A}$ ). Furthermore, the mRNA level of $I L-6$ was much higher, while the expression of $T N F-\alpha$ and $I L-1 \beta$ was increased at early time points in $N L R C 5^{-/-}$peritoneal macrophages. The induction of IFN- $\beta$ was comparable between $\mathrm{NLRC5}^{-/-}$and WT cells (Figure 5B). These results indicate that unlike in BMDCs, NLRC5 plays a negative regulatory role in $\mathrm{NF}-\kappa \mathrm{B}$ signaling in murine peritoneal macrophages. To further confirm the function of NLRC5 in macrophages, we treated peritoneal macrophages with different TLR ligands and measured cytokine release. We observed significantly higher release of IL-6, but not TNF- $\alpha$, in response to stimulation with TLR ligands from 12 to $24 \mathrm{~h}$ poststimulation in $N L R C 5^{-/-}$peritoneal macrophages, compared to WT cells (Figure 5C and Supplementary information, Figure S5A). Therefore, NLRC5 plays a negative role in regulation of $\mathrm{NF}-\mathrm{kB}$ signaling in peritoneal macrophages. The difference between BMDCs and peritoneal macrophages may be due to the different expression level of NLRC5 (Supplementary information, Figure S4E). Interestingly, we did not observe any difference in IL-1 $\beta$ secretion between $N L R C 5^{-/-}$and WT peritoneal macrophages (Supplementary information, Figure S5B), which is consistent with another report [23]. It should be noted that it appears that loss of NLRC5 may enhance more proinflammatory cytokine release at low doses of stimulation, as large differences in IL-6 and TNF- $\alpha$ production were observed between NLRC5deficient and WT cells when a lower dose of LPS (10 ng/ $\mathrm{ml}$ ) was used (Supplementary information, Figure S6).

To elucidate the role of NLRC5 in type I IFN signaling in peritoneal macrophages, we infected cells with VSV-eGFP, and found that IRF3 phosphorylation occurred by $4 \mathrm{~h}$ postinfection and gradually increased with time in $\mathrm{NLRC5}^{-/}$cells. However, IRF3 phosphorylation was barely detectable until $8 \mathrm{~h}$ postinfection in WT macrophages, and p-IRF3 levels in WT cells were generally lower than in $\mathrm{NLRC5}^{-/-}$cells (Figure 5D). In addition, VSV-eGFP-infected (GFP-positive) cells in $N L R C 5^{-/}$ macrophages were much less than in WT cells (Figure $5 \mathrm{E}$ ), suggesting that $N L R C 5^{-/-}$macrophages show enhanced antiviral immunity compared to WT cells. To further investigate the specificity of the inhibitory function of NLRC5 in type I IFN signaling, we treated peritoneal macrophages with VSV-eGFP, poly(I:C)/LyoVec (intracellular poly(I:C)), poly(I:C) and LPS to activate RIG-I-, MDA5-, TLR3- and TLR4-meidated type I IFN signaling, respectively. IFN- $\beta$ secretion was highly increased in $N L R C 5^{-/-}$cells in response to RIG-I/MDA5 ligands (VSV or intracellular poly(I:C)), but was barely changed when the cells were stimulated with LPS or poly(I:C) (Figure $5 \mathrm{~F}$ ), consistent with our previous findings that NLRC5 mainly interacts with RIG-I/MDA5 to block RNA virusinduced type I IFN signaling [17].

To further confirm the role of NLRC5 in immune response to TLR ligands or virus infection in macrophages, we isolated BMMs and treated the cells with TLR ligands or infected them with VSV or dsRNA. We observed a similar pattern of IKK and MAP kinase activation in 
A

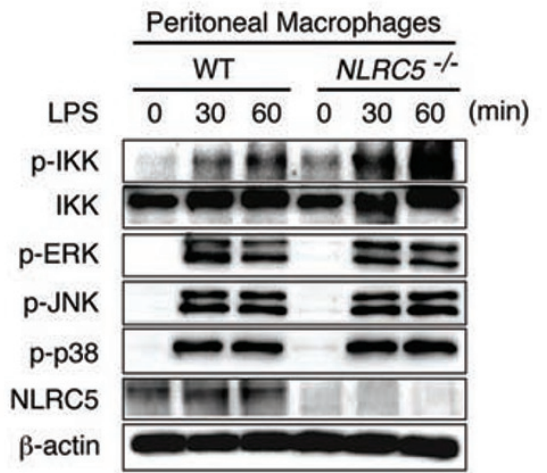

C

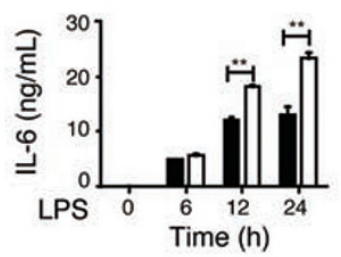

D

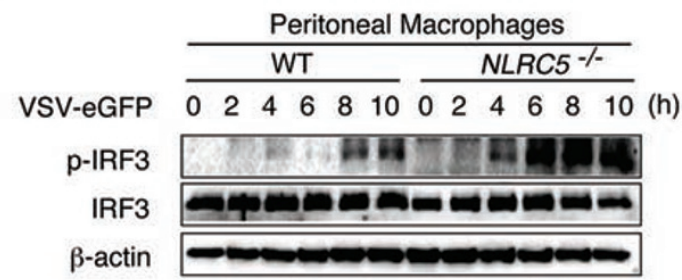

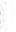

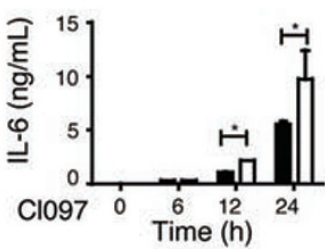

- WT Peritoneal Macrophages

$\triangle$ NLRC5 $\%$ Peritoneal Macrophages
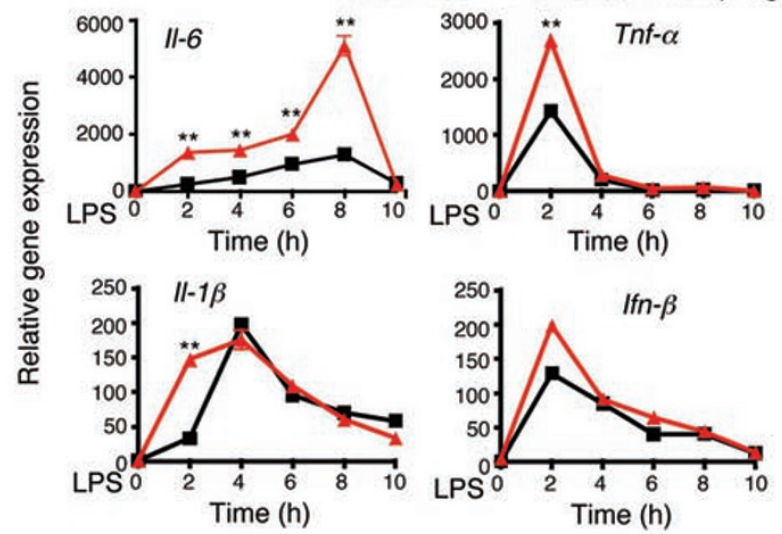
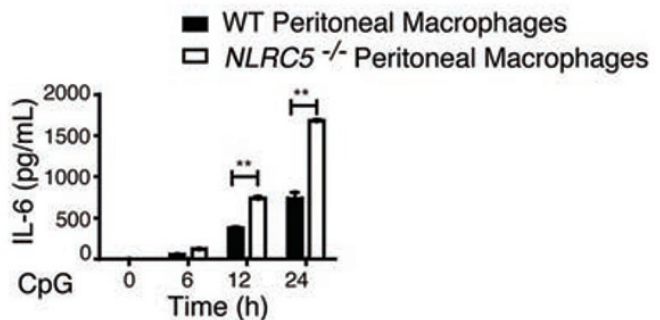

E
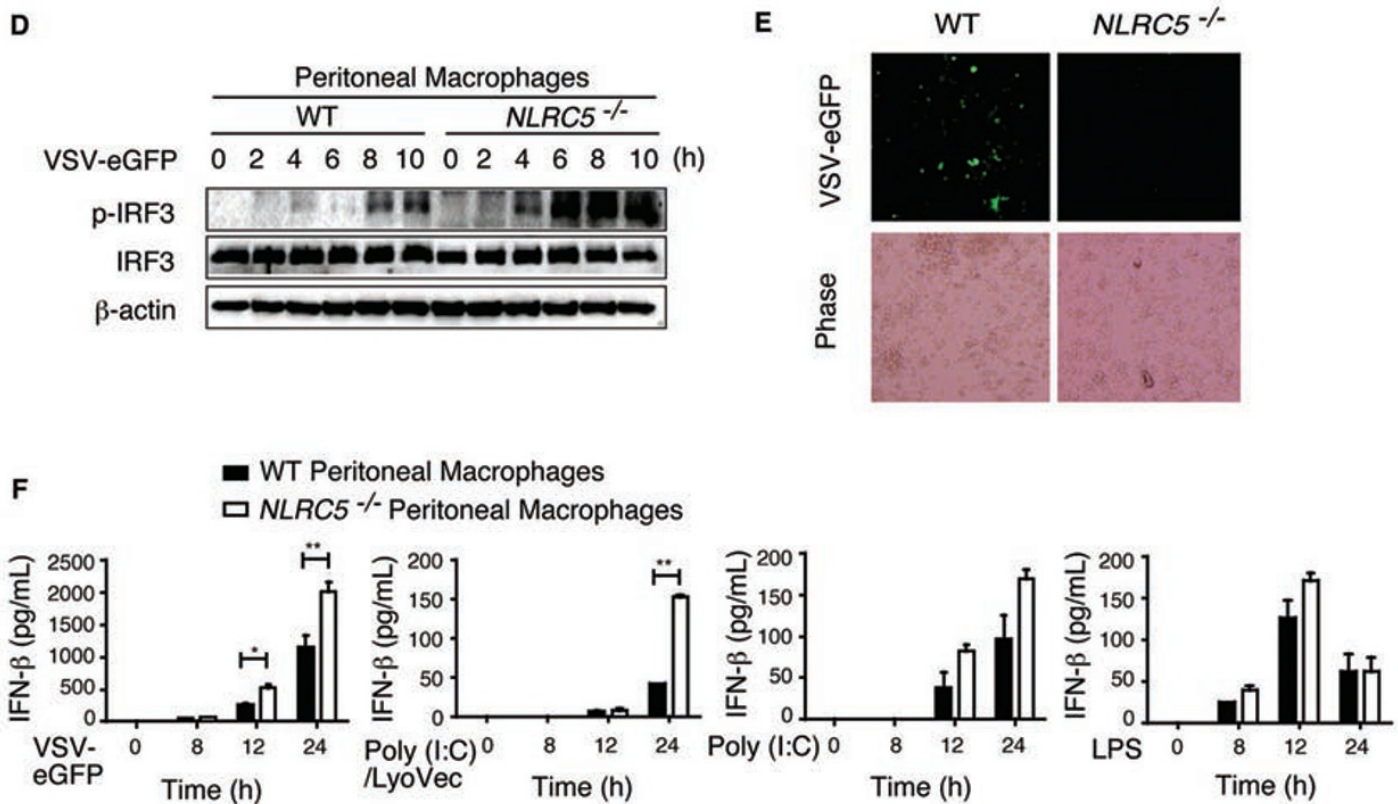

Figure 5 NLRC5 deletion enhances both NF-kB and type I IFN signaling, as well as cytokine production in peritoneal macrophages. (A, B) $N L R C 5^{-1-}$ and WT peritoneal macrophages were incubated with LPS (100 ng/ml) for the indicated times. The cell lysates were harvested for immunoblotting with the indicated antibodies (A). The RNA was collected for real-time PCR analysis (B). (C) NLRC5 $5^{-1}$ and WT peritoneal macrophages were incubated with LPS, CL-097 or CpG for the indicated times, and the culture supernatants were used for ELISA analysis to measure the production of IL-6. (D) NLRC5 ${ }^{-/}$and WT peritoneal macrophages were infected with VSV-eGFP for the indicated times. Cell lysates were harvested to detect IRF3 phosphorylation by immunoblotting. (E) Peritoneal macrophages were infected with VSV-eGFP $(\mathrm{MOI}=10)$ for $24 \mathrm{~h}$, then viral infection was analyzed by fluorescence microscopy (with phase contrast as a control). (F) NLRC5 ${ }^{-1}$ and WT peritoneal macrophages were treated with VSV-eGFP, poly $(\mathrm{I}: \mathrm{C}) / \mathrm{LyoVec}$, poly $(\mathrm{I}: \mathrm{C})$ or LPS for the indicated times, and the culture supernatants were used for ELISA analysis to measure the production of IFN- $\beta$. B, C and $\mathbf{F}$ are plotted as means \pm SD. Results are representative of three independent experiments. ${ }^{*} P<0.05,{ }^{* *} P<0.01$, versus controls. 
$N L R C 5^{-/-}$BMMs to that in peritoneal macrophages (Figure $6 \mathrm{~A}$ ). We also observed that the secretion of IL-6, but not TNF- $\alpha$, was higher in $N R L C 5^{-/}$BMMs compared with WT BMMs, but this difference was not as dramatic as observed in peritoneal macrophages (Figure 6B and Supplementary information, Figure S5C). In addition, we found that phosphorylation of IRF3 was increased after VSV-eGFP infection in $N L R C 5^{-/-}$BMMs compared to WT BMMs (Figure 6C). To further support that NLRC5 inhibits antiviral responses through RIG-I/MDA5 pathways in macrophages, we evaluated the antiviral response after VSV-eGFP, poly(I:C)/LyoVec or poly(I:C) treatment by measuring the IFN- $\beta$ release in culture supernatants. $N L R C 5^{-/-}$BMMs produced more IFN- $\beta$ in response to VSV or poly(I:C)/LyoVec, but not poly(I:C), compared to WT BMMs (Figure 6D). Collectively, these results suggest that NLRC5 ablation enhances both NF- $\kappa \mathrm{B}$ and type I interferon pathways in macrophages.

\section{The function of NLRC5 in inflammation and antiviral im-} munity in vivo

To investigate the role of NLRC5 in LPS-induced septic shock in vivo, we injected $N L R C 5^{-/-}$and WT mice with high-dose Escherichia coli LPS (25 mg/kg) intraperitoneally and then monitored mouse survival. Although we observed a slight, but not statistically significant, difference in survival between the two groups after LPS treatment (Figure 7A), we found a marked increase in plasma IL-6 levels in $N L R C 5^{--}$mice $1 \mathrm{~h}$ after LPS treatment, compared with WT mice (Figure 7B). However, such differences disappeared $3 \mathrm{~h}$ post LPS treatment (data not shown). We did not observe any difference in plasma TNF- $\alpha$ levels (data not shown). These results provide in vivo evidence that NLRC5 negatively regulates IL-6 pro-
A

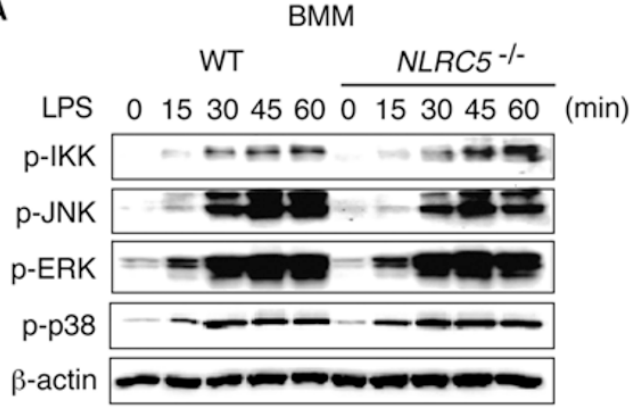

C

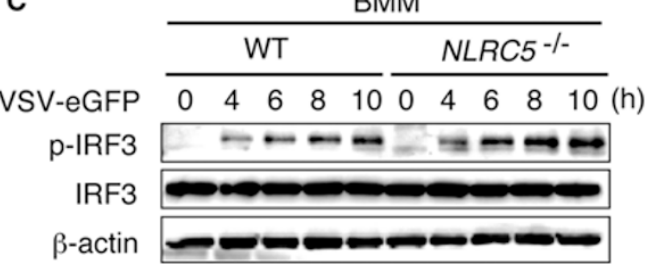

B

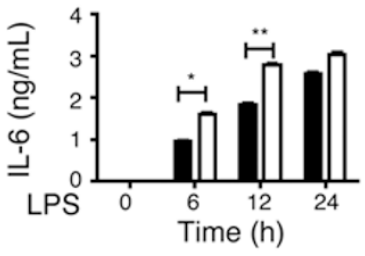

- WT BMM

ㅁ NLRC5 $\%$ BMM

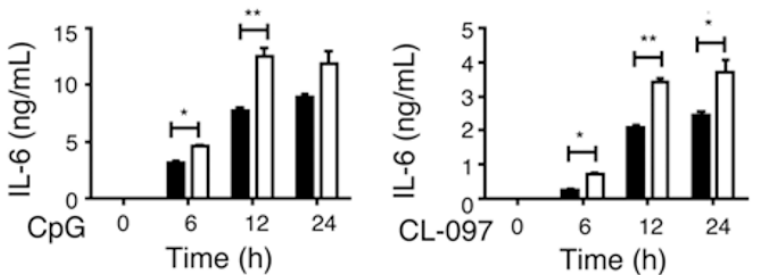

Time (h)

D

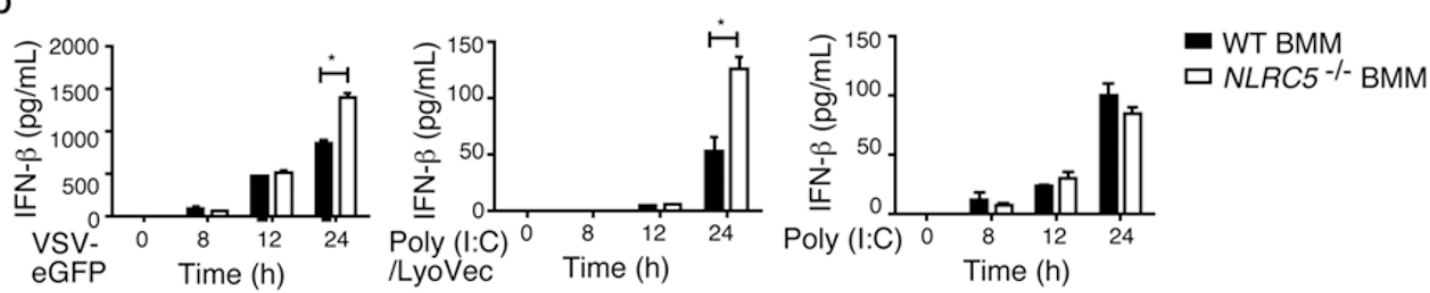

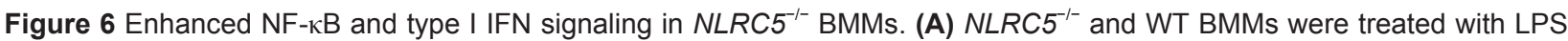
for the indicated times, and the cell lysates were harvested for immunoblotting with the indicated antibodies. (B) BMMs were treated with LPS, CL-097 or CpG for the indicated times, and the culture supernatants were harvested for ELISA analysis. (C) NLRC5 ${ }^{-1-}$ and WT BMMs were infected by VSV-eGFP for the indicated times. Cell lysates were harvested to detect IRF3 phosphorylation by immunoblotting. (D) $N L R C 5^{-1-}$ and WT BMMs were treated with VSV-eGFP, poly(I:C)/LyoVec or poly(I:C) and the culture supernatants were used for ELISA analysis for IFN- $\beta$ production. $\mathbf{B}$ and $\mathbf{D}$ are plotted as means \pm SD. Results are representative of three independent experiments. ${ }^{*} P<0.05$, ${ }^{*} P<0.01$, versus controls. 
A

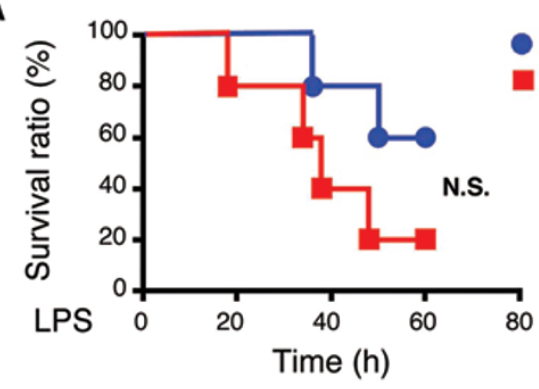

C

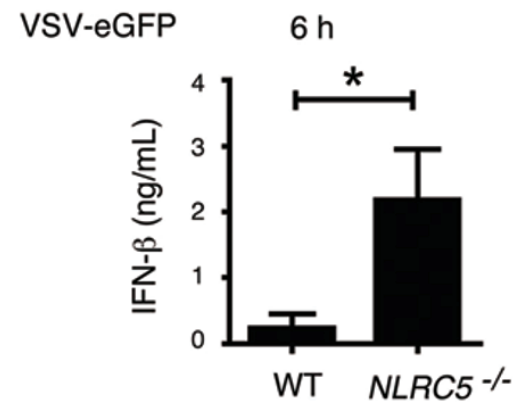

D

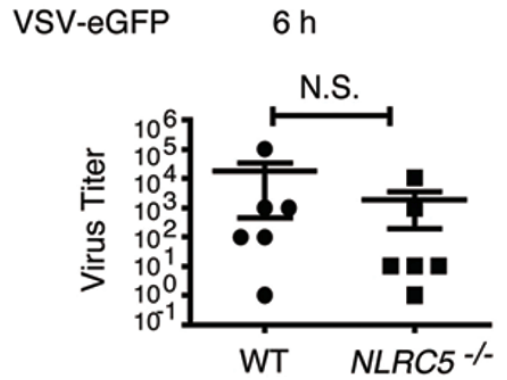

WT

NLRC5 \%
B

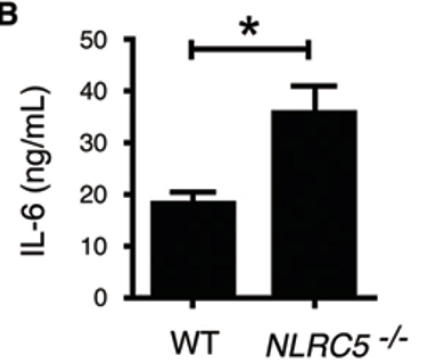

$12 \mathrm{~h}$

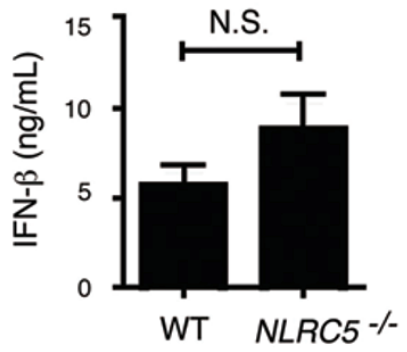

$12 \mathrm{~h}$

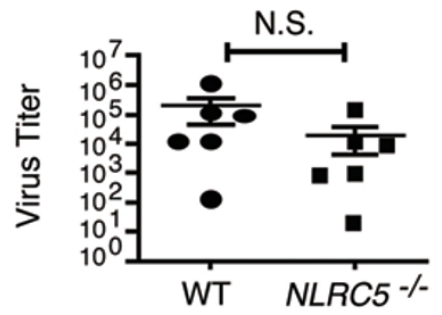

$24 \mathrm{~h}$

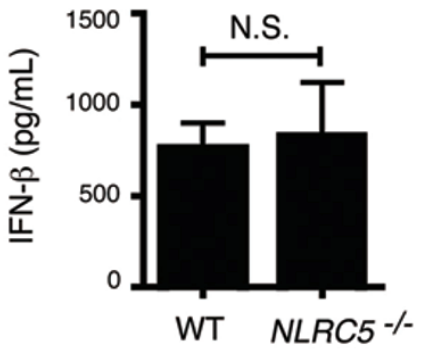

Figure 7 The effect of NLRC5 ablation on LPS-induced septic shock antiviral immunity, and cytokine production in vivo. (A) The survival curve of $N L R C 5^{-1-}$ and WT mice ( $n=5$ per group) after being challenged with LPS (25 mg/kg). (B) Plasma IL-6 levels of WT and $N L R C 5^{-1-}$ mice at $1 \mathrm{~h}$ after peritoneal injection of LPS (25 mg/kg). (C) Plasma IFN- $\beta$ levels at 6,12 and $24 \mathrm{~h}$ after WT and $N L R C 5^{-1-}$ mice ( $n=6$ per group) were injected with VSV-eGFP intraveneously. (D) Plasma virus titration of WT and $N L R C 5^{-1-}$ mice ( $n=6$ per group) at 6,12 and $24 \mathrm{~h}$ after intraveneous injection of VSV-eGFP.

duction at early stages of LPS-induced septic shock, but not at later stages, explaining a slight, but not significant, difference in overall mouse survival after LPS treatment.

We next sought to determine whether NLRC5 deficiency affects antiviral responses in vivo. $N L R C 5^{-/-}$and WT mice were injected with VSV-eGFP via the tail vein and then sera were collected to measure viral titers and type I IFN production. We found a significant increase in IFN- $\beta$ amounts in sera of $N L R C 5^{-/-}$mice compared to WT mice at an early time point $(6 \mathrm{~h})$ postinfection (Figure 7C). However, such differences gradually disappeared at 12 and $24 \mathrm{~h}$ postinfection (Figure 7C). Consistent with this observation, we observed a reduction, but not at significant level, in serum virus titers in $N L R C 5^{-/-}$compared with WT group at 6,12 and $24 \mathrm{~h}$ postinfection (Figure 7D). Consistently, we found that $N L R C 5^{-/-}$mice lived a little longer than WT mice after VSV infection, but not significantly (Supplementary information, Figure S7), suggesting that $N L R C 5$ deficiency increases type I IFN production at early time point in vivo, and antiviral immunity but to a less extent.

\section{Discussion}

Although NLRs were originally identified as intracellular pathogen sensors, recent studies suggest that many NLRs can also function beyond pathogen detection [10]. NLRC5 has been characterized as a regulator of innate 
immunity and MHC class I expression [17-23]. However, the molecular mechanisms and in vivo function of NLRC5 remain to be elucidated. In particular, conflicting roles of NLRC5 in innate immune signaling, antiviral immunity and inflammation have been reported. Thus, it is critically important to further define the function and potential mechanisms of NLRC5 in adverse biological processes. In this study, we generated NLRC5-deficient mice by targeted deletion of the exon 8 of $N L R C 5$, which contains the functional domain of NLRC5 that inhibits $\mathrm{NF}-\mathrm{\kappa B}$ signaling [17], while a previous report characterized mice with targeted deletion of exon 4 of NLRC5. Using newly generated $N L R C 5^{-/-}$mice, we provide several new lines of evidence to elucidate the function and regulatory mechanisms of NLRC5. First, induction of NLRC 5 expression by various TLR and cytokine stimuli is mainly controlled by Stat1-mediated pathway. Stat1 deletion abolished induction of NLRC5 expression by stimulation with LPS or various cytokines, while NF- $\mathrm{BB}$ signaling pathway is required for production of several key cytokines (mainly IFN- $\beta$ ) that in turn activate Stat1mediated signaling, which ultimately control NLRC5 expression. Second, NLRC5 ablation markedly reduced the expression of MHC class I molecule in T cells, as well as in B cells to a less extent, suggesting that NLRC5 plays an important role in the control of MHC class I expression. By contrast, CIITA, another member of NLR protein family, mainly controls MHC class II expression, as well as MHC class I expression in B cells [26, 27]. Third, our results suggest that NLRC5 regulates innate immune responses in a cell type-specific manner. NLRC5 deficiency markedly enhanced NF- $\kappa B$, type I IFN signaling and antiviral immunity in MEFs, macrophages, but not in BMDCs. The cell type-specific effects of NLRC5 on innate immune signaling may be due to the diverse expression levels of NLRC5 in different cell types as previously reported [17-20], and cell type-specific signaling pathways. For example, pDCs predominantly use the MyD88-IRF7 pathway to induce IFN- $\alpha$ production in response to TLR7/9 ligands and viral infection, while other cell types, including macrophages and MEFs, employ TBK1-IRF3-dependent type I IFN signaling in response to RNA/DNA stimulation and viral infection [28]. Our recent studies demonstrate that TGF- $\beta$-activated kinase-1 (TAK1) is an essential positive regulator that triggers NF- $\mathrm{KB}$ and MAP kinase signaling in MEFs and $\mathrm{T}$ cells, but exhibits the opposite effects on these signaling pathways in neutrophils [29]. TRAF family memberassociated NF- $\mathrm{KB}$ activator (TANK) has been identified as a positive regulator of transcriptional factors IRF3 and $\mathrm{NF}-\kappa \mathrm{B}$ signaling. However, a recent study showed that TANK deletion enhanced NF- $\mathrm{KB}$ signaling by promot- ing the ubiquitination of TRAF6 upon TLR stimulation, thus serving as a negative regulator of TLR signaling [30]. In light of these findings, we conclude that NLRC5 functions as a negative regulator of NF- $\mathrm{KB}$ and type IFN signaling in MEFs and macrophages, but has little or no effect on BMDCs.

Kumar et al. [23] generated NLRC5 knockout mice by replacing exon 4 of $N L R C 5$ with a neomycin-resistance gene cassette, and did not observe any effect of NLRC5 deficiency on the induction of inflammatory cytokines and type I IFN in macrophages and dendritic cells (DCs) in response to LPS, poly(I:C), NDV, HSV-1 and $L$. monoctogenes. Our results in BMDCs are consistent with their findings in BMDCs, showing little or no effect of NLRC 5 deletion on innate immune signaling in BMDCs. However, they did not observe any difference between WT and $N L R C 5^{-/}$macrophages in response to LPS or poly(I:C) treatment. The discrepancies between our and their studies may be due to several factors: different deletion mice of NLRC5 (exon 4 versus exon 8 deletion), different doses of LPS used for stimulation and different time courses of TLR stimulation experiments. For example, Kumar et al. used $1000 \mathrm{ng} / \mathrm{ml}$ LPS to stimulate macrophages, which is 10 times higher than the dose that we used $(100 \mathrm{ng} / \mathrm{ml})$. It appears that loss of NLRC5 may enhance more proinflammatory cytokine release at low doses of stimulation, as large differences in IL-6 and TNF- $\alpha$ production were observed between NLRC5deficient and WT cells when a lower dose of LPS (10 ng/ $\mathrm{ml}$ ) was used. High doses of LPS stimulation may mask the differences in IL- 6 and TNF- $\alpha$ production between NLRC5-deficient and WT cells. Moreover, since NLRC5 inhibits NF- $\kappa B$ and type IFN signaling in the early time course [17], we measured innate immune signaling and cytokine production at different time points. More importantly, it should be noted that the differences between NLRC5-deficient and WT cells are observed only when PAMP-mediated signaling activation requires RIG-I/ MDA5 and/or IKK complex. Thus, this specificity may explain why stimulation with poly(I:C), dsDNA, HSV1 and other pathogens showed no difference in cytokine production between NLRC5-deficient and WT cells. In addition, we found that NLRC5 deletion enhanced IL-6 expression in both MEFs and macrophages, but increased production of TNF- $\alpha$ was only observed in NLRC5deficient MEFs but not in macrophages, compared with their WT controls (Figures 3B and 5B). This might be due to multiple layers of regulation for TNF- $\alpha$. It has been known that many cytokine genes such as $T N F-\alpha$ and $I L-1 \beta$ are subjected to posttranscriptional regulation at the mRNA stability and protein translational levels [31]. Consistent with this, we previously showed that 
NLRC5 has more inhibitory effect on IL-6 than TNF- $\alpha$ production in RAW264.7 cells [17]. Similarly, we and others recently found that NLRX1 knockdown or deletion has more dramatic effects on IL- 6 than TNF- $\alpha[12$, 13]. NLRX1 knockdown results in significantly more IL-6 production in the plasma than WT mice in response to LPS treatment, but there is no difference in TNF- $\alpha$ level between NLRX1 knockdown and WT mice. Although most stimuli use the common IKK-NF- $\mathrm{kB}$ module to activate NF- $\kappa B$ signaling, the consequences of activation of downstream NF- $\mathrm{NB}$-responsive genes are often different, mainly due to cell type-specific signaling pathways, quantitative and qualitative parameters of the TLR-ligand interaction itself [32]. Thus, different TLR ligands may use the same NF- $\mathrm{kB}$ pathway, but they may produce different amounts and/or types of cytokines in the same type of cells. Similarly, the same TLR ligand may produce different amounts and/or types of cytokines in different types of cells. More importantly, activation and regulation of the NF- $\kappa$ B pathway itself may vary in a cell type-specific manner. As we recently demonstrated, TAK1 functions as a positive and essential adaptor molecule in NF- $\kappa \mathrm{B}$ and MAP kinase pathways in MEFs, but functions as a negative regulator of these pathways in neutrophils [29]. Thus, it is likely that MEFs use classical NF- $\kappa \mathrm{B}$ pathway, while macrophages and DCs may use the same NF- $\mathrm{BB}$ pathway with even increased variations. Thus, further studies are needed to address cell type-specific activation and regulation of $N F-\kappa B$ and type I IFN signaling pathways.

The functional redundancy of several negative regulators (such as NLRX1 and CUEDC2), which inhibit the same or similar molecules in NF- $\mathrm{KB}$ signaling [12, 33], may compensate for the loss of NLRC5 in innate immune signaling and response to LPS and viral infection in vivo. Our findings show that although NLRC5 deficiency enhanced IL- 6 and IFN- $\beta$ secretion at early time points poststimulation or infection, we observed a small, but not significant, difference in mouse survival after LPS treatment or viral infection. Recent studies indicate that $N L R X 1^{-1-}$ mice exhibited increased expression of IFN- $\beta$ and IL- 6 after influenza virus infection [13], while another group showed no difference in type I IFN signaling between $N L R X 1$-deficient and WT mice [34]. It is not clear what caused these discrepancies between these studies, but it may be due to differences in cell types, duration and dose of stimuli and different experimental system. Alternatively, generation of knockout mice harboring deletions of NLRC5 and NLRX1 may help better understand the role of these negative regulators in vivo.

In summary, we show that induction of NLRC5 expression by various stimuli including LPS and IFN- $\beta$ re- quires Stat1-mediated signaling. NLRC5 ablation reduced MHC class I gene expression in T cells and other cells, suggesting the involvement of NLRC5 in MHC class I expression. NLRC5 ablation enhanced NF- $\mathrm{BB}$ and type I IFN signaling pathways in response to LPS or VSV infection in a cell type-specific manner. $N L R C 5$-deficient mice produced higher amounts of IL- 6 and IFN- $\beta$ in the sera when they were challenged with LPS or infected with VSV. NLRC5 plays important roles in diverse biological processes and immune responses to pathogens in a cell type-specific manner, thus serving as important targets for modulating innate immune signaling and regulation.

\section{Materials and Methods}

\section{Generation of NLRC5-deficient mice}

The targeting vector was constructed by replacing exon 8 , which encodes part of the LRR1 domain of Nlrc5, with a Neo cassette, leading to premature termination of NLRC5 (Figure 1A). The linearized targeting vector was injected into ES cells by electroporation. Homologous recombinant stem cells were identified by screening with PCR and further confirmed by Southern blotting analysis. The selected homologous recombinant ES clones were injected into blastocysts. Chimeric mice generated from two homologous ES clones were bred to generate F1 heterozygotes as well as homozygous NLRC5-deficient mice. All the mice were maintained in a pathogen-free animal facility, following the Use of Laboratory Animals and the approved protocols by the IACUC committee at Baylor College of Medicine.

\section{Cell culture and TLR stimulation}

RAW264.7 cells were purchased from ATCC (Manassas, VA) and maintained in DMEM with 10\% fetal bovine serum (FBS). MEFs were prepared from day 15 embryos and cultured in DMEM supplemented with $10 \%$ FBS. MAVS-deficient MEFs were previously described [12]. Peritoneal macrophages were harvested from mice 4 days after thioglycollate (BD, Sparks, MD) injection and were cultured in DMEM supplemented with $10 \%$ FBS. Bone marrow cells were isolated from the tibia and femur and cultured in RPMI1640 medium with 10\% FBS, 1\% penicillin-streptomycin, $55 \mu \mathrm{M} \beta$-mercaptoethanol and 10\% L929 conditioned media containing macrophage-colony stimulating factor (M-CSF) for 4 days or $10 \mathrm{ng} / \mathrm{ml}$ murine GM-CSF (PeproTech) for 6-8 days to harvest BMMs or BMDCs, respectively. BMMs and BMDCs were stimulated for the indicated times with LPS (100 ng/ml), Pam3CSK4 (1 $\mu \mathrm{g} / \mathrm{ml}), \mathrm{CpG}(2 \mu \mathrm{g} / \mathrm{ml}), \mathrm{CL}-097(1 \mu \mathrm{g} / \mathrm{ml})$, poly(I:C) $(20 \mu \mathrm{g} / \mathrm{ml})$, and poly(I:C)/LyoVec $(1 \mu \mathrm{g} / \mathrm{ml})$, unless specifically mentioned. LPS (E. coli, sterile serotype 0.111 :B4) was purchased from Sigma-Aldrich. CpG ODN 1668, poly(I:C) and other TLR ligands were obtained from Invivogen (San Diego, CA, USA).

\section{Flow cytometry}

Fluorescence-conjugated antibodies against mouse CD4, CD8 and $\mathrm{H} 2-\mathrm{k}^{\mathrm{b}}$ were used in this study. All antibodies were obtained from BD Pharmingen (San Jose, CA, USA). Cells were stained, washed, resuspended in $\mathrm{PBS}(1 \%) \mathrm{FBS}(0.05 \%) \mathrm{NaN}_{3}$ and analyzed 
using a FACSCalibur flow cytometer. All flow cytometry data were analyzed using FlowJo (TreeStar, Inc., Palo Alto, CA, USA).

\section{Antibodies}

Anti-IKK (sc-7607) and anti-IRF3 (sc-9082) were purchased from Santa Cruz Biotechnology (Santa Cruz, CA, USA); anti- $\beta$ actin was purchased from Sigma. Anti-phospho-IKK (2697), antiphospho-ERK (9101), anti-phospho-p38 (9211) anti-phosphoJNK (9251) and anti-phospho-IRF3 (4947) were purchased from Cell Signaling Technology (Danvers, MA, USA). Anti-NLRC5 polyclonal antibody was previously described [17]. Anti-IFN- $\beta$ antibody (7F-D3) was purchased from Abcam. Anti-IFN- $\gamma$ antibody was obtained from clone HB170 (ATCC).

\section{Immunoblot analyses}

For immunoblot experiments, whole-cell extracts were lysed, boiled for $5 \mathrm{~min}$ with SDS loading buffer (Cell Signaling Technology, Danvers, MA, USA) and resolved on SDS-PAGE gels. The proteins were transferred to nitrocellulose membranes (Bio-Rad), blocked with milk and further incubated with the indicated antibodies. LumiGlo Chemiluminescent Substrate System from KPL (Gaithersburg, MD, USA) was used for protein detection.

\section{Cytokine release analysis}

MEF cells or macrophages were seeded on 24-well plates, treated with indicated stimuli and supernatants were collected and subjected to analysis with commercial ELISA kits for mouse IL-6, TNF- $\alpha$, IL-1 $\beta$ (eBiosceince) and IFN- $\beta$ (PBL Biomedical Laboratories), following the manufacturer's instructions. For in vivo LPS septic shock experiments, mice were injected with LPS $(25 \mathrm{mg} /$ $\mathrm{kg})$ intraperitoneally, and sera were collected before $(0 \mathrm{~h})$ and after ( 1 and 3 h) LPS injection and analyzed for their IL-6 and TNF- $\alpha$ levels by ELISA. For in vivo virus infection, mice were injected intravenously with VSV-eGFP. Sera were collected before $(0 \mathrm{~h})$ and after $(3$ and $6 \mathrm{~h}$ ) viral infection and analyzed for the IFN- $\beta$ levels using ELISA.

\section{Real-time PCR analysis}

Total RNA was harvested from MEFs or macrophages using the TRIzol reagent (Invitrogen) and the complimentary cDNA was generated using reverse transcriptase II (Invitrogen). Real-time PCR was carried out using the ABI Prism 7000 analyzer (Applied Biosystems) using the SYBR GreenER qPCR Super Mix Universal (Invitrogen) and specific primers. The sequences of the primers used are:

Primers for mouse Nlrc5: forward: 5'-TCAGCCCAGAACAAGTATCC-3'; reverse: 5'-TGGGCACAGAC-TTCCATTAG-3'.

Primers for mouse Gapdh: forward 5'-TTGTCTCCTGCGACTTCAACAG-3'; reverse: 5'-GGTCTGGGATGGAAATTGTGAG-3'.

Primers for mouse $T N F-\alpha$ : forward: 5'-ACAGAAAGCAT-

GATCCGCG-3'; reverse: 5'-GCCCCCCATCTTTTGGG-3'.

Primers for mouse $I L-6$ : forward: $5^{\prime}$-CCAGAAACCGCTATGAAGTTCC-3'; reverse: 5'-TTGTCACCAGCATCAGTCCC-3'. Primers for mouse $I L-1 \beta$ : forward: 5'-GTGGCTGTGGAGAAGCTGTG-3'; reverse: 5'-GAAGGTCCACGGGAAAGACAC-3'.

Primers for mouse $I F N-\beta$ : forward: 5'-TCACCTACAGGGCG-
GACTTC-3'; reverse: 5'-TCTCTGCTCGGACCACCATC-3'.

\section{Statistical analysis}

Statistical significance between groups was determined by twotailed Student's $t$-test and two-way ANOVA test. Differences were considered to be significant when $P<0.05$. For mouse endotoxic shock study, Kaplan-Meier survival curves were generated and analyzed for statistical significance with Graphpad Prism 4.0.

\section{Acknowledgments}

We would like to thank Dr Adebusola A Ajibade (The Methodist Hospital Research Institute) for critical reading of this manuscript, and Drs Margaret A Goodell and Katherine Yudeh King (Baylor College of Medicine) for providing us with Stat ${ }^{-/}$mice. This work was in part supported by Grants from National Institutes of Health (NIH) (CA090327, CA101795, CA121191, CA116408, CA094327 and DA030338), Cancer Research Institute, and The Methodist Hospital Research Institute. Yanzheng Tong was a recipient of The China Scholarship Council (CSC).

\section{References}

1 Takeuchi O, Akira S. Pattern recognition receptors and inflammation. Cell 2010; 140:805-820.

2 Schroder K, Tschopp J. The inflammasomes. Cell 2010; 140:821-832.

3 Kawai T, Akira S. The role of pattern-recognition receptors in innate immunity: update on Toll-like receptors. Nat Immunol 2010; 11:373-384.

4 Wilkins C, Gale M Jr. Recognition of viruses by cytoplasmic sensors. Curr Opin Immunol 2010; 22:41-47.

5 Chiu YH, Macmillan JB, Chen ZJ. RNA polymerase III detects cytosolic DNA and induces type I interferons through the RIG-I pathway. Cell 2009; 138:576-591.

6 Ablasser A, Bauernfeind F, Hartmann G, Latz E, Fitzgerald KA, Hornung V. RIG-I-dependent sensing of poly(dA:dT) through the induction of an RNA polymerase III-transcribed RNA intermediate. Nat Immunol 2009; 10:1065-1072.

7 Zhang Z, Yuan B, Bao M, Lu N, Kim T, Liu YJ. The helicase DDX41 senses intracellular DNA mediated by the adaptor STING in dendritic cells. Nat Immunol 2011; 12:959-965.

8 Unterholzner L, Keating SE, Baran M, et al. IFI16 is an innate immune sensor for intracellular DNA. Nat Immunol 2010; 11:997-1004.

9 Inohara N, Nunez G. NODs: intracellular proteins involved in inflammation and apoptosis. Nat Rev Immunol 2003; 3:371382.

10 Kufer TA, Sansonetti PJ. NLR functions beyond pathogen recognition. Nat Immunol 2011; 12:121-128.

11 Moore CB, Bergstralh DT, Duncan JA, et al. NLRX1 is a regulator of mitochondrial antiviral immunity. Nature 2008; 451:573-577.

12 Xia X, Cui J, Wang HY, et al. NLRX1 negatively regulates TLR-induced NF-kappaB signaling by targeting TRAF6 and IKK. Immunity 2011; 34:843-853.

13 Allen IC, Moore CB, Schneider M, et al. NLRX1 protein attenuates inflammatory responses to infection by interfering with the RIG-I-MAVS and TRAF6-NF-kappa B signaling 
pathways. Immunity 2011; 34:854-865.

14 Tattoli I, Carneiro LA, Jehanno M, et al. NLRX1 is a mitochondrial NOD-like receptor that amplifies NF-kappa B and JNK pathways by inducing reactive oxygen species production. EMBO Rep 2008; 9:293-300.

15 Jounai N, Kobiyama K, Shiina M, Ogata K, Ishii KJ, Takeshita F. NLRP4 negatively regulates autophagic processes through an association with Beclin1. J Immunol 2011; 186:1646-1655.

16 Fiorentino L, Stehlik C, Oliveira V, Ariza ME, Godzik A, Reed JC. A novel PAAD-containing protein that modulates NF-kappa B induction by cytokines tumor necrosis factoralpha and interleukin-1 beta. J Biol Chem 2002; 277:3533335340 .

17 Cui J, Zhu L, Xia XJ, et al. NLRC5 negatively regulates the NF-kappa B and type I interferon signaling pathways. Cell 2010; 141:483-496.

18 Benko S, Magalhaes JG, Philpott DJ, Girardin SE. NLRC5 limits the activation of inflammatory pathways. J Immunol 2010; 185:1681-1691.

19 Kuenzel S, Till A, Winkler M, et al. The nucleotide-binding oligomerization domain-like receptor NLRC5 is involved in IFN-dependent antiviral immune responses. J Immunol 2010; 184:1990-2000.

20 Neerincx A, Lautz K, Menning M, et al. A role for the human nucleotide-binding domain, leucine-rich repeat-containing family member NLRC5 in antiviral responses. $J$ Biol Chem 2010; 285:26223-26232.

21 Davis BK, Roberts RA, Huang MT, et al. Cutting edge: NLRC5-dependent activation of the inflammasome. J Immunol 2011; 186:1333-1337.

22 Meissner TB, Li A, Biswas A, et al. NLR family member NLRC5 is a transcriptional regulator of MHC class I genes. Proc Natl Acad Sci USA 2010; 107:13794-13799.

23 Kumar H, Pandey S, Zou J, et al. NLRC5 deficiency does not influence cytokine induction by virus and bacteria infections. J Immunol 2011; 186:994-1000.

24 O'Shea JJ, Gadina M, Schreiber RD. Cytokine signaling in 2002: new surprises in the Jak/Stat pathway. Cell 2002; 109 Suppl:S121-S131.

25 Shuai K, Liu B. Regulation of JAK-STAT signalling in the immune system. Nat Rev Immunol 2003; 3:900-911.

26 LeibundGut-Landmann S, Waldburger JM, Krawczyk M, et al. Mini-review: specificity and expression of CIITA, the master regulator of MHC class II genes. Eur J Immunol 2004; 34:1513-1525.

27 Wright KL, Ting JP. Epigenetic regulation of MHC-II and CIITA genes. Trends Immunol 2006; 27:405-412.

28 Honda K, Taniguchi T. IRFs: master regulators of signalling by Toll-like receptors and cytosolic pattern-recognition receptors. Nat Rev Immunol 2006; 6:644-658.

29 Ajibade AA, Wang Q, Cui J, et al. TAK1 negatively regulates NF-kappaB and p38 MAP kinase activation in Gr-1+CD11b+ neutrophils. Immunity 2012; 36:43-54.

30 Kawagoe T, Takeuchi O, Takabatake Y, et al. TANK is a negative regulator of Toll-like receptor signaling and is critical for the prevention of autoimmune nephritis. Nat Immunol 2009; 10:965-972.

31 Han J, Ulevitch RJ. Limiting inflammatory responses during activation of innate immunity. Nat Immunol 2005; 6:11981205.

32 Kanzler H, Barrat FJ, Hessel EM, Coffman RL. Therapeutic targeting of innate immunity with Toll-like receptor agonists and antagonists. Nat Med 2007; 13:552-559.

33 Li HY, Liu H, Wang CH, et al. Deactivation of the kinase IKK by CUEDC2 through recruitment of the phosphatase PP1. Nat Immunol 2008; 9:533-541.

34 Rebsamen M, Vazquez J, Tardivel A, Guarda G, Curran J, Tschopp J. NLRX1/NOD5 deficiency does not affect MAVS signalling. Cell Death Differ 2011; 18:1387.

(Supplementary information is linked to the online version of the paper on the Cell Research website.) 